\title{
Psoriasis at Ayder referral hospital among patients attending dermatology clinic, Mekelle, north Ethiopia
}

\author{
Sintayehu Worku Aynalem ${ }^{1, ~ *, ~ W o r k a l e m a h u ~ A l e m u ², ~ A l e m a y e h u ~ B a y r a y ~}{ }^{3}$, Tilahun Belete Mossie ${ }^{4}$ \\ ${ }^{1}$ Mekelle University, Post Graduate in Dermatology, Mekelle, North Ethiopia \\ ${ }^{2}$ Mekelle University, Dermato-Venerology Department, North Ethiopia \\ ${ }^{3}$ Mekelle University, Public Health Department, North Ethiopia \\ ${ }^{4}$ Mekelle University, School of Nursing, North Ethiopia
}

\section{Email addresses:}

worku.sinayehu@yahoo.com (S. W. Aynalem),mdwork2012@gmail.com (W. Alemu), alemayehub35@gmail.com (A. Bayray), tilahunbe100@gmail.com (T. B. Mossie)

\section{To cite this article:}

Sintayehu Worku Aynalem, Workalemahu Alemu, Alemayehu Bayray, Tilahun Belete Mossie. Psoriasis at Ayder Referral Hospital among Patients Attending Dermatology Clinic, Mekelle, North Ethiopia. Science Journal of Clinical Medicine. Vol. 3, No. 6, 2014 , pp. 106-110. doi: $10.11648 /$ j.sjcm.20140306.12

\begin{abstract}
Background: Psoriasis is a chronic recurrent inflammatory skin condition. The prevalence of psoriasis varies in different population from $0.1 \%$ to $11 \%$. It can classify in to type one ( $<40 \mathrm{yrs}$ ) and type two ( $>=40 \mathrm{yrs})$. And also classified into four major categories; chronic plaque psoriasis, guttate psoriasis, pustular psoriasis and erthroderma. The objective of this study was to assess magnitude and factors associated with psoriasis. Methods: A cross-sectional study design and convenient sampling technique was implemented at Ayder referral hospital dermatologic unit until sample size (422) was fulfilled. Data was entered and analyzed by SPSS version 16.0. Descriptive statistics and logistic regression analysis was done. . The magnitude of psoriasis was $5.4 \%$ where majorities (81\%) of psoriasis cases were chronic plaque. Multiple site involvement $(42.9 \%)$ (arms, trunk and leg) were the main characteristics. Marital status (widowed/divorced) (AOR= 7.75, 95\%CI $(1.58$, $37.98)$ ) and complaining joint pain $(\mathrm{AOR}=7.66,95 \% \mathrm{CI}(2.24,26.17))$ were significantly associated with psoriasis. Conclusion: The magnitude of psoriasis in ARH was relatively higher. Having history of joint pain and being divorced/ widowed were significantly associated with occurrence of psoriasis. Therefore, early diagnosis and treatment of joint pain, minimizing social stressor conditions such as divorce and providing emotional and psychological support were recommended.
\end{abstract}

Keywords: Psoriasis, Ayder Referral Hospital, Dermatologic Clinic

\section{Introduction}

Psoriasis is a chronic inflammatory and recurrent condition of skin (1). The prevalence of psoriasis varies in different population from $0.1 \%$ to $11 \%$ according to published reports (2). Psoriasis is uncommon in West African countries such as Nigeria - 0.8\% (3), Ghana - 0.4\% (4), 3.15\% Kenya (5). There are two different forms of psoriasis exist: type I psoriasis, with age onset of before 40 years, and type II, with age onset of after 40 years (2).

Study conducted in Taiwan showed that (prevalence $=0.235 \%$; mean age $=46.4 \pm 18.6$; male: female $=1.6: 1$ ) and $17.5 \%$ of cases were sPsO type (34).Other study in Taiwan showed that prevalence of psoriasis was $0.23 \%$ for men and $0.16 \%$ for women, respectively. The prevalence of psoriasis increased more rapidly in male patients aged 30 years. (7).

Study done in Norway prevalence of was $1.4 \%$, with no difference between males and females. Peak prevalence was observed in the age group 30. A higher prevalence of psoriasis among females was found in urban and higher prevalence in females. No difference in prevalence was found between various socio-economic groups (8).Other study at dermatology Clinic in a General Hospital in Port-ofSpain, Trinidad and Tobago, three hundred and seventy-nine cases $(5.1 \%$ of the total cases) of psoriasis was diagnosed. There were 183 females $(48 \%)$ and 196 males (52\%). The mean age at presentation for females was 44 years and that for males was 43 years. The majority of cases (77\%) presented between 20-69 years (9). 
Population-based survey conducted in China at six cities, out of 17,345 subjects showed that 102 subjects $(0.59 \%)$ were found to have psoriasis. The prevalence of psoriasis in males and females were $0.54 \%$ and $0.44 \%$ respectively. Psoriasis vulgaris accounts $97.06 \%$ and family history of psoriasis accounted (28.43\%) (10). Community based study conducted at Japan showed that shows males $(65.8 \%)$ and females $(34.2 \%)$. The vast majority of cases $(86.0 \%)$ had plaque-form of psoriasis vulgaris, and 812 cases $(2.8 \%)$ were guttate psoriasis. Psoriatic erythroderma $(0.8 \%)$, generalized pustular psoriasis $(0.9 \%)$, and localized pustular psoriasis $(0.5 \%)$ were rare (11). Also other Study conducted at dermatology clinic in Hospital Tengku Ampuan Rahimah, Klang Malaysia, and the prevalence of $9.5 \%$ was by psoriasis In this study psoriasis was found to be more common in males' $11.6 \%$ (316/2613) than females $7.2 \%(215 / 2994)$. The most common affected sites were arms (53\%) followed by legs (44\%) and also it involve trunk (15.9\%), genital region $(12.4 \%)$, scalp $(11.8 \%)$, eyebrows $(6.8 \%)$ and face $(2.4 \%)$ (12).

Study conducted in Egypt at Dermatology Clinic, AlHussein University Hospital shows $52.0 \%$ of the cases were females and $44 \%$ of case occur $25-45$ years followed by $37.0 \%$ $(<25 \mathrm{yrs})$ and $19.0 \%>45$ years respectively. The average age of onset was 31 years (13). In other hand since there is limited number of literatures in context of study area. So this study would give base line information about magnitude and factors associated with psoriasis for health professionals and those who are interested in carrying out further research.

\section{Methodology}

\subsection{Study Design and Period}

Institution based cross-sectional study was conducted at Ayder Referral Hospital, Mekelle from July 1 to August 1 2014 about a month using quantitative data collection method.

\subsection{Study Area}

Mekele is the capital city of Tigeray regional state. It is located $730 \mathrm{~km}$ north of Addis Ababa with a total estimated population of 215,914 , of whom 104,925 are men and 110,989 women. The two largest ethnic groups are Tigre (96.2\%), and Amhara (2.26\%) (14).

Ayder referral hospital is a referral and teaching governmental hospitals under the ministry of health. It serves up to 8 million populations in its catchment areas of the Tigray, Afar and South-eastern parts of the Amhara Regional States. It provides a broad range of medical services to both in and out patients of all age groups. As such, the hospital can be designated as the most advanced medical facility, by all accounts, in the Northern part of the country and that it stands as the second largest hospital in the nation. It has 500 inpatient beds in four major departments and other specialty units. It is also used as a teaching hospital for the College of Health Sciences, Mekelle University (15)

\subsection{Population}

\subsubsection{Source Population}

All Clients attending dermatologic clinic at Ayder Referral Hospital.

\subsubsection{Study Population}

All dermatologic patients at dermatology Clinic in Ayder Referral Hospital during Study period.

\subsubsection{Sample Population}

All dermatologic patients at dermatology Clinic in Ayder Referral Hospital during Study period.

Inclusion and exclusion criteria

All clients who visited dermatologic unit, Ayder Referral Hospital during the

Study period except children less than ten (10) years old.

Sample size determination and Sampling technique

\subsection{Sample Size}

The sample size for this particular study was calculated using formula for a single population proportion considering the following assumptions: A 95\% confidence level, margin of error (0.05) and assume maximum variability $(\mathrm{p}=0.5)$. These parameters were substituted in the following single population proportion formula.

$$
\mathrm{n}=\frac{(\mathrm{Z} \alpha / 2) 2 \mathrm{P}(1-\mathrm{P})}{\mathrm{d} 2}
$$

Where:

$\mathrm{n} \quad$ the desired sample size

P Assume maximum variability (50\%).

critical value for normal distribution at $95 \%$ $\mathrm{Z} \alpha / 2$ confidence level which equals to 1.96 ( $\mathrm{z}$ value at $=0.05$ )

D The margin of error taken as 0.05

Therefore, the formula yielded 384 sample size and adding $10 \%$ (38 clients) for non-respondent the total minimum larger sample size is 422 .

\subsubsection{Sampling Technique}

A convenience sampling method was used. All clients attending dermatologic units was interviewed and examined for psoriasis until sample size was fulfilled during data collection period.

\subsection{Study Variables}

Dependent variables: Psoriatic disease

Independent variables: Age, Sex, Marital status, residence, occupation, educational status, ethnicity, religion and average monthly income, family history of psoriasis, obesity, smoking, HIV co-infection, DM, alcohol consumption and URTI.

\subsection{Data Quality Control}

Data collection tools and procedure: Data was collected using structured questionnaire which were adopted from 
different literatures based on objectives of the study. The data was collected by three MSc tropical dermatology students. At the end of the day principal investigator was checked daily activity of data regarding the completion of the questionnaires, clarity of responses and proper coding of the respondents.

Data management and Analysis: The collected data was checked for completeness, range and logical sequence of responses. Data was entered in to SPSS version 16.0 software package for analysis. Binary logistic regression analysis was made to obtain odds ratio and the confidence interval of statistical associations. Those variables with $\mathrm{P}$ value of less than or equal to 0.25 was selected for further analysis. The strength of statistical association was measured by adjusted odds ratios and $95 \%$ confidence intervals and Statistical significance was declared at $(\mathrm{P}<0.05)$.

Operational Definitions: Psoriasis: A skin lesion which is presented with characteristic feature of well demarcation,

adherent silvery scaly and erythematic back ground on the skin or Diagnosed as psoriasis by dermatologist. Psoriatic nail change: Pits range from 0.5 to $2.0 \mathrm{~mm}$ in size, oil spots and salmon patches on the nail of psoriatic patients. Geographic tongue: asymptomatic erythematous patches with serpiginous borders on the tongue.

Ethical consideration: Ethical clearance was obtained from the ethical review committee of the CHS of Mekelle University and it was taken to Tigray Regional Health Bureau. Accordingly, permission letter was secured from medical directors at Ayder Referral Hospital. In addition all of the study participants were informed about the purpose of the study and oral/verbal consent was obtained.

\section{Result}

\subsection{Socio-Demographic Characteristics}

Table 1. Factors associated with psoriasis at Ayder Referral Hospital, OPD, and Aug 2014.

\begin{tabular}{|c|c|c|c|c|c|}
\hline \multirow{2}{*}{ variables } & \multirow{2}{*}{ Category } & \multicolumn{2}{|c|}{ Psoriasis } & \multirow{2}{*}{$\operatorname{COR}(95 \% \mathrm{CI})$} & \multirow{2}{*}{$\operatorname{AOR}(95 \% C I)$} \\
\hline & & Yes & No & & \\
\hline \multirow{2}{*}{ Joint pain } & Yes & 8 & 27 & $7.8(2.98,20.49)$ & $7.66(2.3,26.17)^{*}$ \\
\hline & No & 13 & 343 & 1.0 & 1.0 \\
\hline \multirow{2}{*}{ Alcohol use } & Yes & 6 & 44 & $2.96(1.09,8.08)$ & $2.02(0.59,6.89)$ \\
\hline & No & 15 & 326 & 1.0 & 1.0 \\
\hline \multirow{3}{*}{ HIV status } & Positive & 4 & 20 & $3.5(1.04,11.76)$ & $1.58(0.19,7.2)$ \\
\hline & Not known & 4 & 122 & $0.58(0.18,1.8)$ & $0.74(0.22,2.54)$ \\
\hline & Negative & 13 & 228 & 1.0 & 1.0 \\
\hline \multirow{3}{*}{ Marital status } & Divorced/widowed & 7 & 17 & $9.4(2.94,30.04)^{*}$ & $7.75(1.58,37.98)^{*}$ \\
\hline & Not married & 7 & 193 & $0.83(.28,2.41)$ & $1.4(0.41,4.81)$ \\
\hline & Married & 7 & 167 & 1.0 & 1.0 \\
\hline \multirow[t]{2}{*}{ Age } & $<40$ & 15 & 326 & 1.0 & 1.0 \\
\hline & $\geq 40$ & 6 & 44 & $2.96(1.09,8.04) *$ & $1.34(0.30,6.02)$ \\
\hline \multirow[t]{2}{*}{ Occupation } & Unemployed & 4 & 27 & $2.98(0.98,9.5)$ & $1.13(0.26,9.4)$ \\
\hline & Employed & 17 & 343 & & 1.0 \\
\hline
\end{tabular}

$\mathrm{COR}=$ Crude odds ratio, $\mathrm{AOR}=$ Adjusted odds ratio, $*$ significantly associated

From the total of 422 identified dermatologic cases at dermatologic OPD, 391 were included in the analysis making the response rate of $92.64 \%$. The mean $( \pm \mathrm{SD})$ age of the respondent was $27.5( \pm 12.5)$ years. One hundred ninety seven $(50.4 \%)$ were females while $353(90.3 \%)$ of the respondents were orthodox by religion. The largest ethnic group was Tigrie $362(92.6 \%)$. Concerning the educational status of patient, $83.6 \%$ had attended formal school out of which $33.8 \%$ had accomplished above twelve. Nearly half of patients were not married; students and government employee account $32.2 \%$ and $24.6 \%$ respectively. Nearly two third $(71.4 \%)$ of them were urban by place of residence. Out of the total Study subjects (39.9\%) had no income and $33.2 \%$ of them earn an average monthly income of greater than or equal to 1200 ETB (Table: 1).

\subsection{Clinical Characteristics of Psoriasis}

Out of the total study participants, $21(5.4 \%)$ of the patient were psoriatic (male; $61.9 \%$ Vs females; $38.1 \%$ ). The mean $( \pm \mathrm{SD})$ age of the psoriatic patient was $35.4( \pm 14.6)$ years. Also the duration of lesion less than five years accounts $61.9 \%$ which was similar to the lesion recurrent. Pruritus of the lesion was $85.7 \%$ out of this moderate pruritus accounts $42.8 \%$ followed by sever pruritus $28.6 \%$. Eighteen $(85.7 \%$ ) from total of psoriatic patient had erythematous back ground with 95.2\% whitish/slivery scales. Additional physical findings presented with psoriasis such as nail pitting, geographic tongue, and salmon patch which accounts 52.9\%, 42.9\% \& $19 \%$ respectively. Nearly four fifth $(81 \%)$ of psoriasis clinical type was chronic plaque followed by ertheroderma 9.5\%. Mostly (42.9\%) involved sites were multiple site, palm and sole $23.8 \%$. In addition less than $10 \%$ body surface was accounts $47.6 \%$. Above half (52.4\%) of psoriatic patient didn't know aggravating factors but seasonal variation contributes to $28.6 \%$. Two third of patient were on follow up and the treatment modality was topical and systemic $42.9 \%$ and $23.8 \%$ respectively. Four of them had other medical problem with three of them on other medication.

\subsection{Clinical and Behavioral Related Factors}

Out of study subject $2(.5 \%)$ of them had family related psoriasis. Joint pain accounts nearly one tenth 35 (9\%), while 
common cold like symptom accounts $16(4.1 \%)$. And alcohol drinkers, cigarette smokers, known HIV and known DM were $50(12.8 \%), 9(2.3 \%), 25(2.4 \%)$, and $9(2.35)$ respectively. More than half 234 (59.95) of study participants had body mass index of less than twenty.

\subsection{Factors Related to Psoriasis}

The bivariate logistic regression shows that among the socio-demographic characteristics age and marital status were significantly associated with psoriasis; in which age of 40 years or older and divorced or widowed were risk factors for psoriasis. Also from clinical and behavioral factors those who have joint pain, alcohol drinkers and known HIV positives were risk factors for psoriasis

\subsection{Multivariate Analysis}

To identify factors associated with psoriasis, multivariable logistic regression analysis was carried out using variables in the binary logistic regression with a p-value $\leq 0.25$. Therefore, the multivariable logistic regression analysis, marital status and joint pain were significantly associated with psoriasis. Hence, those who were divorced/widowed were about 8 times more likely to develop psoriasis than married people $(\mathrm{AOR}=7.75,95 \% \mathrm{CI}(1.58,37.98))$. In addition those who had joint pain were about eight times more likely to develop psoriasis than those who had no joint pain (AOR=7.66, 95\%CI $(2.24,26.17)$ ) (Table: 1$)$

\section{Discussion}

Psoriasis is a global problem; its magnitude varies worldwide from $0.24 \%$ at Tiwan (6), $0.4 \%$ at Ghana (4), 0.59 at china (10), $0.8 \%$ at Nigeria (3), 3.1\% at Kenya (5), $5.1 \%$ at Trinidad and Tobago (9) with $9.5 \%$ at Malaysia (12). Similar research was done at Black lion hospital, Ethiopia reported that prevalence of psoriasis accounts $2.25 \%$ (16), also in this study the prevalence was determined to be $5.4 \%$ which was higher than Tiwan, Ghana, Nigeria ,china, Kenya and Black lion studies but it was similar to Trinidad and Tobago finding. Even though it was lower than the Malaysian study. The possible reasons could be that lower prevalence of researches was done at community level other than Black Lion study. The socio-demographic variation might have contributed for the discrepancy with that of the Black lion finding. There was a difference in study design with that of Malaysian; retrospective study design was conducted for three years in Malaysian study while in this study cross-sectional study design was implemented. Regarding to clinical characteristics of psoriasis a study conducted at Egypt reported that the mean age of onset was 31 years (13); whereas the Malaysian research found out that most of the case of psoriasis lay in the age group of 40-60 years (12). Also in this study the mean age of onset was 35 years which almost similar to that of Egypt. While more than two-third $(71 \%)$ of the cases were less than 40 years old unlike Malaysian finding. It might be the delay to treatment at Malaysia for the difference. On the other hand studies done at Trinidad and Tobago and at Japan showed that more than half of the psoriatic cases were males $(9,11)$. But another study done at Egypt reported that $52 \%$ cases were females (13). In this study males accounted $62 \%$ which is similar to the Japan and Trinidad and Tobago findings unlike Egypt study. Concerning comparison between urban and rural areas higher prevalence was found in urban areas at Norway (8) which is similar to this study by which three fourth of psoriatic cases were from urban places.

As regards family history of psoriasis, it is $14 \%$ and $41 \%$ if one and both parent/s are affected respectively (2). Community based study conducted at China reported that family history of psoriasis accounted $28 \%$ (10), but in this study it was $9.5 \%$ from psoriatic cases. The variation could be due to a difference in study design. There are different clinical types of psoriasis, from these types psoriasis vulgaris was the commonest type at China and Japan $(10,11)$ which is similar to this study which accounted $81 \%$. Concerning the degree of psoriasis, sever type was found in $17.5 \%$ and $26 \%$ of the cases at Taiwan and Egypt respectively $(6,13)$; while in this study it was $28.6 \%$ which relatively higher than the former studies, the possible reasons might be low level of awareness about the disease, poor adherence and delay in treatment. The sites highly affected by psoriasis include arms, legs and trunk in Malaysian study (12); in similar fashion multiple sites (arms, legs and trunk (43\%)) followed by palm and sole $(24 \%)$ were sites affected in current study.

Marital status was significantly associated with psoriasis; thus divorced and widowed people were nearly eight times more likely to develop psoriasis than married people $(\mathrm{AOR}=7.75,95 \% \mathrm{CI}(1.58,37.98))$. This might be related to psychological stress among divorced and widowed individuals. The relation of rheumatoid arthritis and psoriasis is the erosive inflammatory arthritis that is at the root of the cause for both conditions. They also share a gene called Runx 1 which is a protein that acts as a transcription factor. When Runx1 changes; it contributes to autoimmune disorders like psoriatic arthritis and rheumatoid arthritis (17). Beside above, joint pain was other significantly associated with psoriasis, which were those people who had joint pain were about eight time at risk of developing psoriasis than those who had no joint pain ( $\mathrm{AOR}=7.66,95 \% \mathrm{CI}$ (2.24, 26.17)). The possible reason might be joints are targets of immune attack so that one of the possible pathogenesis of psoriasis is immunology.

\section{Conclusion and Recommendation}

\subsection{Conclusion}

The magnitude of psoriasis at Ayder Referral Hospital was found to be attention seeking. Fortunately, this study has assessed the magnitudes of eczema, fungal infection and other dermatologic conditions which have found to be more prevalent. Even if different researches have reported that age, income and other socio-economic status and environmental 
condition were factors for psoriasis, in this study sociodemographic (marital status), clinical (joint pain) were identified factor of the occurrence of psoriasis.

\subsection{Recommendation}

To regional health bureau and stakeholder

- Should strengthen its attention for dermatologic diseases control while designing and implementing health programs and strategies

To dermatologists and clinicians

- Early diagnosis and treatment of joint pain should be emphasized

- Educate the patients to consult the health professionals when they feel joint pain

- Should give emphasis to treat and prevent eczema and fungal infection

To the community and community leaders

- Minimizing the chance of divorce and strengthening marital life should be enhanced

- Provide emotional and psychological support to divorced and widowed individuals to decrease stress

To researcher

To assess the factors associated with psoriasis using stronger study design

\section{Acknowledgment}

I would like to Mekelle University health Science College and Dermato-venorology Department for giving the golden opportunity just for learn more. Also my thanks go to data collectors and study participants without them it is impossible to chive.

\section{References}

[1] Rosa P, Deborah P, Christopher E, et al. Global Epidemiology of Psoriasis. J Investigative Dermatology 2012; 133:377.

[2] Wolf G, Barbara A, David J. Psoriasis. In: klaus W, Lowell A, Goldsmith Stephen I (eds). Fitzpatrick's Dermatology in General Medicine. England; McGraw-Hill 2008, 170-193.
[3] Obasi O. Psoriasis vulgaris in the Gunea Savannah region of Nigeria. Int J Dermatol 1086; 25:181-3.

[4] Doe P, Aseidu A, Acheampong J, Rowland P. Skin diseases in Ghana and the UK. Int J Dermatol 2001; 42: 323-6.

[5] Leder R, Farber E. The variable incidence of psoriasis in subSaharan Africa. Int J Dermatol 1997; 36: 911-9.

[6] Tsai T,Wang T, Hung S,et al. Epidemiology and comorbidities of psoriasis patients in a national database in Taiwan. J Dermatol Sci 2011 Jul; 63(1):40-6.

[7] Chang Y, Chen T, Liu P, et al. Epidemiological study of psoriasis in the national health insurance database in Taiwan. Acta Derm Veereonl 2009; 89(3):262-6.

[8] Braathen L, Botten G, Bjeerkedal T. Prevalence of psoriasis in Norway. Acta Derm Venereol Suppl 1989; 142:5-8.

[9] Suite M. Epidemiology of Psoriasis in a Dermatology Clinic in a General Hospital in Port-of-Spain, Trinidad and Tobago, West Indies. West Indian Med J 2006; 55 (6): 1

[10] Ding X, Wang T, Shen Y, et al. Prevalence of psoriasis in China, a population-based study in six cities. Eur J Dermatol 2012; 22(5):663-7.

[11] Kawada A, Tezuka T, Nakamizo Y, Kimura H, et al. A survey of psoriasis patients in Japan from 1982 to 2000. J Dermatol Sci 2003 Feb; 31(1):59-64.

[12] Sinniah B. Epidemiology of Psoriasis in Malaysia. Med J Malaysia June 2010; 65:2

[13] Essam A. Ibrahim S, Hamed O, et al. psoriasis and psoriatic arthritis: characteristics and risk factors among adult patients in Egypt. Egyptian Journal of Hospital Medicine April 2012; 47: $301-320$.

[14] Official Website Mekele City. URL: www.mekellecity.com /accessed at May 2014.

[15] Official website of Mekele University. URL: www.mu.edu.et / accessed at May 2014.

[16] Shibeshi D. Pattern of skin diseases at the University Teaching Hospital, Addis Ababa, Ethiopia. Int J Dermatol 2000; 39: 822-825. www.arthritis.com/ accessed at Aug 2014/ 